\title{
La motivación en el aprendizaje de las matemáticas con Pizarra Digital Interactiva
}

\section{Motivation in learning mathematics with Interactive Whiteboard}

\author{
María Cancelo*, Francisco Caamaño** \\ *IES Universidade Laboral, **Universidade de A Coruña
}

\begin{abstract}
Resumen
La intención de este estudio ha sido conocer el nivel de motivación de los estudiantes en el aprendizaje de las matemáticas con pizarra digital interactiva (en adelante PDI). Se han impartido dos temas con una misma metodología, uno con y otro sin PDI, en dos grupos de primero de ESO. Los resultados de nuestra investigación reflejan una débil motivación respecto al aprendizaje de matemáticas con PDI en las dimensiones analizadas.

Palabras clave: pizarra digital interactiva, matemáticas, motivación, TIC
\end{abstract}

\begin{abstract}
The purpose of this research has been to know the motivational level of students in the learning of mathematics when an interactive whiteboard is used (IWB). Two first level groups of ESO have been taught by using the same methodology applied to two lessons: one of the groups drew on IWB, the other one had to do without it. The survey results hint a weak motivation in the analysed dimensions. Keywords: interactive whiteboard, IWB, motivation, ICT
\end{abstract}

\section{Introducción}

Siguiendo los estudios de Weiner (1985) sobre aprendizaje y motivación, el aprendizaje varía en función de los diferentes grados o niveles de motivación del alumno, por lo que cabe deducir que ambos aspectos están íntimamente relacionados: lo cognitivo va intrínsecamente unido a lo emocional (Barberá, 1997; Dweck, 1986), mientras que el aprendizaje está caracterizado como un proceso cognitivo y motivacional a la vez (González-Cabanach, Barca, Escoriza y González, 1996).

Se trata, por tanto, de aprovechar esa relación para propiciar un aprendizaje significativo que tenga en cuenta el interés, afición, afán de superación, de adquirir competencias, habilidades, etc. por parte del alumno, factores estos que, entre otros, constituyen la dimensión "motivación" en el aprendizaje.

En la actualidad, debido a la introducción en las aulas de las nuevas tecnologías de la información, el alumno está expuesto a nuevos estímulos, mientras que los profesores tienen la oportunidad de ofrecer también nuevos enfoques en sus clases utilizando esta tecnología. Las TIC se están mostrando como un recurso educativo potente y las formas de trabajo en la educación están cambiando, tanto en lo concerniente al aprendizaje como a la enseñanza. Es previsible, por tanto, que este nuevo escenario ejerza alguna influencia sobre la motivación del alumnado (Coll, 2004; Suárez, 2010).

Nosotros nos vamos a centrar precisamente en el aspecto motivacional del aprendizaje bajo el prisma de una de las herramientas tecnológicas que está irrumpiendo con fuerza en las aulas: la PDI (Betcher y Lee, 2009; Derek y Miller, 2001; Marqués, 2010, 2012).

Concretamente, este trabajo está dirigido al estudio de la percepción del alumno sobre su motivación en el aprendizaje de las matemáticas con PDI. Se trata de obtener datos que nos ayuden a comprender cómo percibe el alumno su motivación tras haber introducido en el proceso de enseñanza-aprendizaje una PDI.

El estudio se ha llevado a cabo en las aulas Abalar de un centro de educación secundaria de la provincia de A Coruña, en dos clases de primer curso de educación secundaria en la clase de matemáticas.

Se trata de un estudio cuantitativo con un diseño cuasiexperimental. Se ha trabajado con dos grupos (un grupo de control y un grupo experimental) a los que se les ha entregado un cuestionario tipo Likert basado en ítems contrastados en distintos estudios sobre la motivación, que se refieren a las distintas dimensiones de esta. Los resultados obtenidos han sido tratados estadísticamente para extraer las conclusiones del estudio.

Si bien existen otros estudios sobre el empleo didáctico de esta herramienta por el profesorado (Betcher y Lee, 2009; Cole, 2010; Morgan, 2008), así como sobre las necesidades formativas que esta requiere para un aprovechamiento real (Beeland, 2002; Gallego, Cacheiro y Dulac, 2009; Marqués, 2010; Prats, Riera, Gandol y Carrillo, s.f.) este estudio intenta aportar nuevos datos concretos a este panorama general, estudiándolo desde el punto de vista del alumno, en particular en lo concerniente al grado de motivación que el estudiante percibe cuando la PDI es utilizada en la clase de matemáticas (Miller, Glover y Averis, 2004).

\section{Método}

Este trabajo se dirige a dilucidar la cuestión de si los alumnos perciben una mayor motivación en el 
aprendizaje cuando se emplea como herramienta de trabajo la PDI. Se trata de un estudio cuantitativo descriptivo tipo survey, transversal, realizado a través de un cuestionario proporcionado a una muestra de alumnos de primer ciclo de ESO en la asignatura de matemáticas. El diseño es cuasi-experimental, ya que no incluye el uso de una asignación aleatoria, sino que los grupos ya están organizados antes del experimento. La muestra está formada por dos grupos del mismo nivel, con edades y rendimiento similares, constituidos con un criterio que no afecta al estudio (apellido y elección de una optativa) y no hay indicios de que se haya producido ningún hecho que pueda influir en los resultados. Así pues, los grupos pueden considerarse equiparables en la variable de nuestro estudio (Amal, del Rincon y Latorre, 1992; Fraenkel, Wallen y Hyun, 2011; Hernández, Fernández y Baptista, 1997).

Tal como hemos mencionado, existen varios trabajos en profundidad sobre la visión y empleo de esta herramienta por el profesorado, así como sobre el factor motivador de las TIC en general; sin embargo, este estudio intenta mejorar nuestro conocimiento sobre el tema aportando datos extraídos de los propios alumnos, específicamente sobre la PDI y centrados en la motivación. Para la definición de motivación remitimos a las siete dimensiones de motivación de Passey (2004), que comprenden: interés en progresar, autopercepción de eficacia en el uso de las TIC, reconocimiento del valor de uso, motivación intrínseca (entendida como la capacidad de las TIC de enganchar y mantener el interés del alumno), búsqueda de mejora del propio desempeño, medio de recompensa, condicionamientos externos y falta de motivación. Exponemos más adelante, en el apartado de "resultados", el análisis de los datos obtenidos en cada una de estas dimensiones.

Por tanto, nuestro trabajo quiere conocer el nivel de motivación de los alumnos cuando se emplea la pizarra digital como herramienta didáctica en el aula. De esta forma, se podrían ofrecer evidencias sobre una mayor, menor o nula diferencia de motivación en estas circunstancias.

Se eligieron para el estudio dos clases con un mismo profesor que utiliza las mismas estrategias didácticas. Es este profesor el que imparte los dos temas del experimento. Cada grupo es grupo de control y experimental en dos exposiciones diferentes a la variable independiente (PDI).

Para comprobar la homogeneidad de los grupos se pasó un pretest previo a la exposición de los alumnos al tratamiento (PDI sí o no) en el que se recaban datos significativos de los mismos.

Los grupos, experimental y de control, fueron expuestos a la explicación de dos temas, números enteros y números decimales, así como al desarrollo de las distintas actividades. En ambas fases, el grupo de control no contó con el apoyo de la PDI y el grupo experimental sí lo tuvo. Al finalizar el primer tema, se pasó un cuestionario tipo Lickert a todos los estudiantes. Se explicó a continuación un segundo tema y se desarrollaron los distintos ejercicios y actividades. El grupo de control del primer tema pasó a ser el grupo experimental en este segundo tema y el grupo experimental pasó a ser grupo de control. Al finalizar el tema los estudiantes volvieron a cubrir de nuevo el cuestionario para mantener los resultados consistentes.

Los métodos de análisis de datos utilizados responden al tipo de investigación que se trata, de tipo cuantitativo: frecuencias, porcentajes y medias. Para el tratamiento de datos se utiliza la hoja de cálculo Excel y el paquete estadístico SPSS.

\section{Participantes}

Como hemos dicho anteriormente, se trabajó con alumnos de primer curso de ESO de un instituto de A Coruña, elegido al azar, que está incurso en el proyecto Abalar. Hay que destacar también que los grupos son equivalentes tanto al inicio del experimento como durante su desarrollo.

Por último, es el mismo profesor el que imparte clase en ambos grupos, utilizando una misma forma didáctica:

Para el grupo de control, el método tradicional (explicación del tema con la pizarra tradicional, ejercicios escritos relacionados con el tema y corrección de los mismos).

Para el grupo experimental, el uso de la pizarra digital, sin dejar por ello de trabajar también con el método tradicional.

El experimento se aplicó durante medio trimestre y el profesor explicó un tema en cada fase. Nada más finalizar cada tema, el profesor pasó los cuestionarios a ambos grupos.

\section{Instrumentos}

Previo a las sesiones de clase se pasó un pretest para recoger información descriptiva de los alumnos participantes en el estudio y poder garantizar la homogeneidad de los grupos.

Tras las sesiones de clase con y sin PDI, se suministra un postest. Se trata de un cuestionario ex post facto donde se plantean al alumno cuestiones relativas a las dimensiones estudiadas, que en nuestro caso son: interés en progresar, eficacia académica, reconocimiento del valor de uso, motivación intrínseca, medio de recompensa, condicionamientos externos, y falta de motivación.

Este instrumento consta de una serie de ítems que recogen información sobre cómo perciben los estudiantes el uso o la ausencia de PDI en el aula. La escala utilizada es de tipo Likert de cinco niveles, codificando las respuestas en: totalmente en desacuerdo $=1$, desacuerdo $=2$, neutral $=3$, de acuerdo $=4$, muy de acuerdo $=5$.

El cuestionario que se ha utilizado ha sido elaborado a partir del utilizado por Passey (2004) en su investigación y del inventario de motivación intrínseca -IMI(Monteiro, Mata y Peixoto, 2015).

\section{Procedimiento}

El trabajo de campo consiste en la exposición a la PDI, en distintos periodos de tiempo, de dos grupos de primer ciclo de secundaria de aulas Abalar, de características similares en cuanto a edad, rendimiento, etc. Es un mismo profesor el que, utilizando la misma forma didáctica, desarrolla dos temas de matemáticas en cada una de las aulas y solo en uno de ellos se utilizará la PDI 
como herramienta didáctica. El período total de tiempo es de 6 semanas, distribuidas en periodos de 3 semanas cada uno.

Se les pasaron a los dos grupos de estudiantes en las distintas fases del experimento los 23 ítems de la escala Likert para recoger sus percepciones. Ambos grupos dispusieron del mismo tiempo para cubrir el cuestionario y se les dieron las mismas indicaciones. Es el profesor el encargado de entregar y recoger los cuestionarios a los alumnos.

Se ha elegido este tipo de escala porque es utilizada para medir actitudes mediante una serie de ítems presentados como aseveraciones, que el encuestado ha de valorar en distinto grado (Hernández, Fernández y Batista, 1997). Se ha puesto especial cuidado en la redacción de la escala para que esta contenga ítems valorados tanto directa como inversamente, de forma que exija al alumno una reflexión atenta para su contestación y nos sirva para comprobar la coherencia de las respuestas a la hora del análisis de resultados.

Los datos recogidos de los cuestionarios se analizan de forma independiente para cada una de las etapas, para posteriormente sacar una conclusión conjunta.

En un primer momento se analizan los resultados del pretest. Estos resultados son tratados con el programa SPSS y mediante un análisis comparativo de los distintos apartados que figuran en el pretest se llega o no a la conclusión de si los dos grupos son homogéneos. En caso de no tener grupos homogéneos se hace una nueva selección de los grupos hasta asegurar la semejanza necesaria para que los resultados obtenidos sean representativos.

La siguiente fase en la estrategia analítica consiste en analizar los resultados del postest para cada una de las siete dimensiones aquí estudiadas, comparando los resultados entre el grupo experimental y el grupo de control. Para cada dimensión se hace una media de los resultados para una posterior comparación de medias entre los dos grupos.

\section{Resultados}

Los resultados de nuestra investigación reflejan que los estudiantes se perciben un poco más motivados cuando se utiliza la PDI. De todos modos, las diferencias en ambas fases entre el grupo de control y el experimental son muy pequeñas $y$, a la vista de los estudios estadísticos, no se pueden considerar significativas.

$\mathrm{Se}$ han medido las distintas dimensiones motivacionales de los alumnos con y sin PDI en la clase de matemáticas y los resultados aparecen como sigue:

\section{Interés en progresar}

Los alumnos del grupo experimental demuestran un mayor interés en progresar que los alumnos del grupo de control, en ambas fases, por lo que podría colegirse que el uso de la PDI hace que el alumno participe en la actividad porque muestra interés en mejorar tanto la comprensión como la capacidad, pero las pruebas estadísticas no corroboran esta suposición.

\section{Eficacia académica}

De nuevo en ambas fases los alumnos del grupo experimental mejoran en resultados a los alumnos del grupo de control, aunque las diferencias son pequeñas. La prueba estadística de la $T$ de Student nos indica que la PDI no tiene un efecto significativo sobre la capacidad que cree tener el alumno para alcanzar un objetivo mediante la PDI.

\section{Reconocimiento del valor de uso}

Es en la primera fase donde se aprecia una diferencia entre la puntuación del grupo experimental y de control que nos hace pensar que el alumno reconoce ventajas propias de la PDI cuando le ofrecen la posibilidad de su empleo y, aunque en la segunda fase las diferencias entre el grupo experimental y el de control son muy pequeñas, los resultados estadísticos nos indican que en esta dimensión sí es significativa la diferencia entre el uso y no uso de la PDI.

\section{Motivación intrínseca}

Las bajas puntuaciones obtenidas en esta di-mensión en ambos grupos y en ambas fases, no nos indican que la PDI haya captado al alumno ni que haya conservado su interés.

\section{Medio de recompensa}

En esta dimensión se muestran bajos niveles de puntuación, lo cual es deseable, aunque es en el grupo experimental donde se obtienen unos resultados ligeramente superiores a los del grupo de control. De todos modos, debido al valor tan bajo de la media podemos concluir que el alumno no se interesa en el uso de la PDI como recurso para conseguir retroalimentación positiva.

\section{Condicionamientos externos}

Aquí los resultados están por encima del 3 en ambos grupos y en las dos fases lo que puede indicar que el alumno se implica no motu proprio, sino porque se siente de alguna forma condicionado por figuras externas, pero no se aprecian diferencias entre el grupo que usa PDI y el que no la usa.

\section{Falta de motivación}

En esta dimensión hay diferencias entre el grupo de control y el experimental. Las mayores puntuaciones del grupo de control parecen indicar que cuando no se usa la PDI hay un número mayor de alumnos que no se implican en la tarea y no muestran indicio alguno de interés, aunque la prueba de comparación de Kruskal Wallis no aprecia diferencias significativas.

A la vista de los resultados anteriores, parece que tan solo hay pequeñas diferencias a favor del grupo experimental en las dimensiones interés en progresar, eficacia académica, motivación intrínseca y falta de motivación y solo se han encontrado diferencias significativas en la dimensión "reconocimiento del valor de uso". No se evidencia, por tanto, una relación entre el uso de la PDI y la motivación, puesto que en ninguna de las dimensiones analizadas, salvo en una, se han encontrado diferencias significativas entre el grupo que hizo uso de la PDI y el que no lo hizo.

Concluimos por lo tanto que tan solo el uso de la PDI en el aula puede no ser suficiente para influir 
significativamente en la motivación de los alumnos. Sería necesario un estudio de mayor calado sobre los usos que los docentes dan a esta herramienta, para saber si el verdadero potencial de la PDI, su capacidad para conjugar visualización e interactividad, se está explotando; a la vez será importante estudiar el uso de esta herramienta con una enseñanza centrada en el estudiante.

\section{Referencias}

Arnal, J., del Rincón, D., Latorre, A. (1992). Investigación educativa. Fundamentos y metodología. Barcelona: Labor.

Barberá, E. (1997). Modelos explicativos en psicología de la motivación. REME, 4(10). Recuperado de http://reme.uji.es/articulos/abarbe7630705102/texto.ht $\mathrm{ml}$.

Beeland, W. (2002). Student Engagement, Visual Learning and Technology: Can Interactive Whiteboards Help? Recuperado de http://downloads.smarttech.com/media/research/intern ational_research/usa/beeland_am.pdf.

Betcher, C. y Lee, M. (2009). The interactive whiteboard revolution: teaching with IWBs. Australia: ACER Press. Recuperado de http://schoolnet.org.za/CoL/ACE/course/classroom/do cuments/iwb_revolution.pdf.

Cole, J. D. (2010). The impact of interactive whiteboards on students' attitudes toward applied mathematics (Tesis doctoral, Universidad de Nipissing, Canada). Recuperado de http://search.proquest.com/docview/814798803?accou ntid=15299.

Coll, C. (2004). Psicología de la educación y prácticas educativas mediadas por las tecnologías de la información y la comunicación: una mirada constructivista. Sinéctica. 25, 1-24. Recuperado de http://www.redalyc.org/html/998/99815899016/.

Derek, G., Miller, D. (2001). Running with technology: the pedagogic impact of the large-scale introduction of interactive whiteboards in one secondary school. Journal of Information Tecnology for Teacher $\begin{array}{llll}\text { Education, } & 10 & (3), & 257-278 .\end{array}$ 10.1080/14759390100200115.

Dweck, C. S. (1986). Motivational processes affecting learning. American Psychologist, 41 (10), 1040-48. Recuperado http://s3.amazonaws.com/academia.edu.documents/25 951781/motivational_processes.pdf?AWSAccessKeyI d=AKIAIWOWYYGZ2Y53UL3A\&Expires $=149979$ 0378\&Signature $=$ RLtFOwP3lqbfutKFOwPGkyqRIZk \%3D\&response-content-

disposition=inline $\% 3 \mathrm{~B} \%$ 20filename $\% 3$ DMotivational _processes_affecting_learnin.pdf.

Fraenkel, J., Wallen, N., \& Hyun, H. (2011). How to design and evaluate research in education. New York: McGraw-Hill.

Gallego, G., Cacheiro, M. L., Dulac, J. (2009). La pizarra digital interactiva como recurso docente. Teoría de la Educación: Educación y Cultura en la Sociedad de la $\begin{array}{llll}\text { Información. } 10 & \text { (2). Recuperado de }\end{array}$ http://www.redalyc.org/html/2010/201017352009/.

González-Cabanach, R., Barca, A., Escoriza, J. y González, J. (1996) Psicología de la instrucción: aspectos históricos explicativos y metodológicos. Barcelona: E.U.B.

Hernández, R., Fernández, C. y Baptista, P. (1997). Metodología de la investigación. México: McGrawHill.

Marqués, P. (2010). Investigación / Plan de formación: centros de excelencia Smart 2008-2010 (Web) Recuperado de http://www.peremarques.net/smart/.

Marqués, P. (2012). La pizarra digital (web). Recuperado de http://www.peremarques.net/pizarra.htm.

Miller, D., Glover, D. y Averis, D. (2004). Motivation: The contribution of interactive whiteboards to teaching and learning in mathematics. Recuperado de http://rcsdk8.edlioschool.com/pdf/technology_commit tees/iwb/IWB_MOtivation.pdf.

Monteiro, V., Mata, L. y Peixoto, F. (2015). Intrinsic Motivation Inventory: Psychometric Properties in the Context of First Language and Mathematics Learning. Psicologia: Reflexão e Crítica, 28(3), 434-443. Doi:10.1590/1678-7153.201528302.

Morgan, G. L. (2008). Improving student engagement: Use of the interactive whiteboard as an instructional tool to improve engagement and behavior in the junior high school classroom (Tesis doctoral, Universidad de Liberty, Virginia, USA). Recuperado de http://digitalcommons.liberty.edu/cgi/viewcontent.cgi ?article $=1140 \&$ context $=$ doctoral.

Passey, D., Rogers, C., Machell, J. y McHugh, G. (2004). The motivational effect of ICT on pupils. Department of Educational Research. UK: Universidad de Lancaster. Recuperado de http://downloads01.smarttech.com/media/research/int ernational_research/uk/lancaster_report.pdf.

Fernández, M., i Romaní, J., Casado, F. y Álvarez, E. (2012). Autopercepción y demandas del profesorado de infantil y primaria sobre formación en pizarra digital interactiva. Pixel-Bit. Revista de Medios y Educación, 40, 89-100. Recuperado de https://idus.us.es/xmlui/bitstream/handle/11441/45685 /file_1.pdf?sequence $=1 \&$ isAllowed=y.

Suárez, C. (2010). La formación en red como objeto de estudio (en línea). RUSC. Revista de Universidad y Sociedad del Conocimiento, 7(2). Barcelona: UOC. Recuperado https://dialnet.unirioja.es/descarga/articulo/3664428.p df.

Weiner, B. (1985). An attributional theory of achievement motivation and emotion. Psychological Review, 92(4), 548-573. Recuperado de http://acmd615.pbworks.com/f/weinerAnattributionalt heory.pdf. 